\title{
Resposta clínica e metabólica de potros neonatos em relação aos achados histopatológicos da placenta na égua
}

[Clinical and metabolic response of neonatal foals related to histopathology finds in mare placenta]

L.A. Lins $^{1}$, I.S. Finger ${ }^{2}$, C.G. Fernandes ${ }^{3}$, B.R. Curcio $^{3}$, M.N. Corrêa ${ }^{3}$, C.E.W. Nogueira ${ }^{3}$

\author{
${ }^{1}$ Faculdade de Veterinária - Universidade da Região da Campanha - URCAMP - Bagé, RS \\ ${ }^{2}$ Aluna de graduação - Faculdade de Veterinária - Universidade Federal de Pelotas - Pelotas, RS \\ ${ }^{3}$ Faculdade de Veterinária - Universidade Federal de Pelotas - Pelotas, RS
}

\begin{abstract}
RESUMO
Avaliaram-se as respostas clínica e metabólica de potros neonatos em relação aos achados histopatológicos da placenta na égua. Foram avaliados dois grupos de éguas da raça Puro Sangue Inglês um grupo-problema $(n=25)$ e um grupo-controle $(n=25)$, de acordo com os achados da placenta. $O$ exame dos potros constou de avaliação clínica geral, hematologia e bioquímica sérica. $\mathrm{O}$ exame histopatológico da placenta apresentou resultado compatível com a apresentação clínica do potro, sendo que a presença de lesões inflamatórias resultou na produção de potros debilitados. A presença de lesões degenerativas não comprometeu o estado clínico do neonato, mas pode ser responsável pela manifestação de distúrbios subclínicos, evidenciados pelo aumento das taxas de AST e GGT. A ureia pareceu ser um indicador de dano renal decorrente de insuficiência placentária em potros neonatos.
\end{abstract}

Palavras-chave: potro, placenta, metabolismo, histopatologia

\begin{abstract}
The placenta represents the major communication between the mare and the fetus during the gestational period, and this suggests that any disturbance in the placenta can be an indicator of gestational damage with risk to the fetus. The aim of this paper was to evaluate the clinical and metabolic responses of the newborn foals related with the findings from the histopathological examination of the placenta. This study was conducted in a farm located in Bagé-RS, Brazil, where were evaluated two groups of Throughbred mares for this case-control study: One Problem Group $(N=25)$ and the Control Group $(n=25)$, based on the placental findings. The foal's evaluation was based on general clinical examination, hematology and serum biochemistry. Results from the placenta histopathological exams were compatible with clinical presentation of the foals, with the presence of inflammatory lesions resulting in the production of debilitated foals. The presence of degenerative lesions in the placenta does not compromise the clinical features of the newborn, but they can be responsible for the manifestation of sub-clinical disturbances, evidenced by increased levels of AST and GGT. Urea seems to be an indicator of renal damage due to placental insufficiency in neonatal foals.
\end{abstract}

Keywords: foal, placenta, metabolism, histopathology

\section{INTRODUÇÃO}

A placenta é um órgão fundamental na relação materno-fetal e deve ser considerada como um reflexo das condições nutricionais, metabólicas, endócrinas e vasculares maternas, além de ser indicativa da condição clínica e metabólica do neonato (Hay Jr., 1995; Jansson e Powell, 2006). A insuficiência placentária resulta no comprometimento das trocas metabólicas e gasosas entre a mãe e o feto (Marconi et al., 1999). Os potros nascidos de uma égua com

Recebido em 17 de agosto de 2011

Aceito em 5 de julho de 2012

E-mail: lucianaalins@yahoo.com.br 
comprometimento placentário podem ser prematuros, com alteração clínica incompatível com a vida, ou ainda podem nascer a termo, com pouca ou nenhuma alteração (Bain, 2004).

A abordagem de um potro de risco deve incluir desde dados do monitoramento da gestação, exame clínico do potro, além de uma avaliação da placenta (Morresey, 2005). O exame da placenta deve ser realizado por meio de avaliação macroscópica e reconhecimento de lesões histopatológicas e é utilizado no diagnóstico de placentite (Hong et al., 1993) ou outras alterações placentárias (Schlafer, 2004)

O objetivo deste estudo foi avaliar as respostas clínica e metabólica de potros neonatos em relação aos achados histopatológicos da placenta na égua, bem como a influência dos distúrbios placentários nos metabolismos fetal e neonatal.

\section{MATERIAL E MÉTODOS}

O estudo foi realizado em um haras de criação do município de Bagé-RS, durante a temporada reprodutiva de 2009. Foram utilizados dois grupos de éguas da raça Puro Sangue Inglês para a realização do estudo de caso - controle: um grupo-problema $(\mathrm{n}=25)$, constituído por éguas que produziram placentas com lesões histológicas, e um grupo-controle $(n=25)$, com éguas que produziram placentas sem alteração. As éguas foram identificadas e acompanhadas durante a gestação até o parto, e os partos foram assistidos para o reconhecimento de qualquer alteração.

A placenta foi inspecionada e pesada imediatamente após a expulsão, sendo considerado também o tempo de eliminação das membranas fetais. Para a avaliação histopatológica, foram coletados fragmentos de sete pontos, considerando-se os dois cornos, corpo, bifurcação, estrela cervical, âmnion e cordão umbilical, conforme o método de Schlafer (2004). Os fragmentos foram fixados em formalina a $10 \%$ em tubos Falcon de $50 \mathrm{~mL}$, garantindo a proporção de 10 partes de solução fixadora para uma parte de tecido. O material clivado foi processado rotineiramente e incluído em blocos de parafina. Cortes de 3 a $5 \mu \mathrm{m}$ foram obtidos, montados sobre lâminas histológicas, desparafinizados, hidratados e corados pelo método de hematoxilina-eosina segundo técnica descrita por Allen (1994). As lâminas foram avaliadas por microscopia óptica.
Os potros foram pesados e submetidos à avaliação clínica geral imediatamente (até uma hora) após o parto. Foi avaliado o comportamento dos potros considerando-se o tempo em que se posicionaram em decúbito esternal, o tempo para reflexo de sucção, para levantar, para a primeira mamada e para a eliminação do mecônio. Previamente à ingestão do colostro, foi coletado sangue com e sem anticoagulante para realização de hemograma e bioquímica sérica, respectivamente.

A análise hematológica foi realizada utilizandose sangue dos potros com EDTA coletado imediatamente após o parto. O hematócrito (HT) foi processado pelo método de micro-hematócrito. As avaliações de proteínas plasmáticas totais (PPT) e de fibrinogênio (FB) foram realizadas por refratometria. A contagem de leucócitos totais (LT) foi realizada em contadora de células automática CELM CC-510, utilizando-se como diluente para as amostras solução hemolisante para contagem de glóbulos brancos, à base de surfactante e cianeto de potássio $(\mathrm{CNK}) 4 \mathrm{mg} / \mathrm{dL}$.

As análises bioquímicas foram realizadas por colorimetria utilizando-se kits comerciais específicos para cada análise. As amostras foram processadas de acordo com as informações do fabricante dos kits, e as leituras realizadas em espectrofotômetro de luz visível Femto - 700 Plus ${ }^{\circledR}$, utilizando-se cubeta de quartzo fosca com parede espessa. O comprimento da onda era ajustado para cada análise, assim como a calibração do espectrofotômetro, que era realizada com o tubo branco específico de cada teste. Foram considerados os níveis das enzimas gama-glutamiltransferase - GGT (Gama GT Liquiform - Labtest $\left.{ }^{\circledR}\right)$ e aspartato aminotransferase - AST (AST/GOT Liquiform Labtest ${ }^{\circledR}$ ); e os valores de ureia (Ureia CE Labtest $\left.{ }^{\circledR}\right)$, creatinina (Creatinina - Labtest $\left.{ }^{\circledR}\right)$ e bilirrubina (Bilirrubina - Labtest ${ }^{\circledR}$ ).

Com base nos achados histopatológicos das placentas, o grupo-problema foi subdividido em grupo-problema inflamatório $(\mathrm{n}=6)$, no qual foram categorizados os potros provenientes de placentas com alterações inflamatórias, e o grupo-problema degenerativo $(\mathrm{n}=19)$, formado por potros nascidos de placentas com alterações degenerativas. 
A análise estatística foi realizada utilizando-se o teste Tukey para comparação de médias entre os grupos, com auxílio do software Statistix 8.0®. Os valores de $\mathrm{P}<0.05$ foram considerados significativos.

\section{RESULTADOS}

Não foram observadas alterações durante os partos. Na avaliação da placenta, o peso médio foi de $6,442 \pm 1,32 \mathrm{~kg} \quad(3,750-9,250 \mathrm{~kg}) . \quad \mathrm{Na}$ avaliação histopatológica, foram encontradas lesões degenerativas, caracterizadas por áreas de degeneração celular do epitélio coriônico e de vilosidades e focos de calcificação sobre o córion, ou alterações inflamatórias evidenciadas por infiltrados celulares mistos, predominantemente histio-linfocitários com presença de alguns neutrófilos. Foram consideradas alteradas as placentas que apresentaram duas ou mais porções com lesões degenerativas sem a presença de reação inflamatória, ou apenas uma porção com alteração inflamatória.

O peso médio dos potros ao nascer foi de 57,2 $\pm 4,9$ (44-69), não havendo diferença entre o grupo-controle e o grupo-problema $(\mathrm{P}>0,7)$. No entanto, no grupo-problema inflamatório, o peso dos potros foi significativamente menor que $\mathrm{o}$ peso dos potros do controle $(\mathrm{P}<0,05)$. A relação do peso do potro com o da placenta foi de $11 \pm 2,14 \%$ (7-16\%). A média dos pesos dos diferentes grupos está apresentada na Tab. 1.

Tabela 1. Médias e desvio-padrão de peso do potro, peso da placenta e relação entre o peso do potro e da placenta para os quatro grupos, de acordo com os achados histopatológicos da placenta

\begin{tabular}{lccc} 
& $\begin{array}{c}\text { Peso do potro } \\
\mathrm{kg}\end{array}$ & $\begin{array}{c}\text { Peso da placenta } \\
\mathrm{kg}\end{array}$ & $\begin{array}{c}\text { Potro } x \text { placenta } \\
(\%)\end{array}$ \\
\cline { 2 - 4 } & Média* $^{*}$ & Média* $^{*}$ & Média* $^{*}$ \\
\hline Grupo-controle & $56.96 \pm 1.04 \mathrm{a}$ & $6.410 \pm 0.27 \mathrm{a}$ & $11.32 \pm 0.46 \mathrm{a}$ \\
Grupo-problema & $57.39 \pm 1.04 \mathrm{a}$ & $6.470 \pm 0.28 \mathrm{a}$ & $11.50 \pm 0.48 \mathrm{a}$ \\
Grupo-problema inflamatório & $51.75 \pm 2.41 \mathrm{~b}$ & $5.380 \pm 0.63 \mathrm{a}$ & $10.00 \pm 1.61 \mathrm{a}$ \\
Grupo-problema degenerativo & $57.95 \pm 1.12 \mathrm{a}$ & $6.720 \pm 0.31 \mathrm{a}$ & $11.67 \pm 0.49 \mathrm{a}$ \\
\hline
\end{tabular}

*Letras diferentes na coluna significam diferença significativa entre médias.

$\mathrm{Na}$ avaliação do comportamento, foi observado um tempo significativamente maior para ficar em decúbito esternal $(\mathrm{P}<0,01)$ e para levantar $(\mathrm{P}>0,01)$ nos potros do grupo-problema inflamatório. As médias de tempo: ficar em decúbito esternal, apresentar reflexo de sucção, levantar, realizar a primeira mamada e a eliminação de mecônio nos diferentes grupos estão expressas na Tab. 2.

Tabela 2. Médias, desvio-padrão e significância de tempo para decúbito esternal, reflexo de sucção, levantar, primeira mamada e eliminação de mecônio nos diferentes grupos

\begin{tabular}{lcccc}
\multicolumn{1}{c}{ Tempo em minutos } & Grupo-controle & $\begin{array}{c}\text { Grupo- } \\
\text { problema }\end{array}$ & $\begin{array}{c}\text { Grupo-problema } \\
\text { inflamatório }\end{array}$ & $\begin{array}{c}\text { Grupo-problema } \\
\text { degenerativo }\end{array}$ \\
\hline Decúbito esternal & $2.87 \pm 6.91 \mathrm{a}$ & $8.76 \pm 4.74 \mathrm{a}$ & $26.75 \pm 8.78 \mathrm{~b}$ & $3.23 \pm 5.36 \mathrm{a}$ \\
Reflexo de sucção & $33.29 \pm 2.80 \mathrm{a}$ & $30.10 \pm 2.65 \mathrm{a}$ & $24.80 \pm 5.06 \mathrm{a}$ & $32.00 \pm 3.11 \mathrm{a}$ \\
Ato de levantar & $37.17 \pm 5.93 \mathrm{a}$ & $46.10 \pm 5.61 \mathrm{a}$ & $74.60 \pm 9.35 \mathrm{~b}$ & $35.92 \pm 6.52 \mathrm{a}$ \\
Primeira mamada & $50.94 \pm 4.54 \mathrm{a}$ & $55.55 \pm 4.41 \mathrm{a}$ & $52.20 \pm 8.43 \mathrm{a}$ & $56.84 \pm 5.17 \mathrm{a}$ \\
Eliminação do mecônio & $62.20 \pm 4.79 \mathrm{a}$ & $71.27 \pm 4.37 \mathrm{a}$ & $78.80 \pm 8.25 \mathrm{a}$ & $68.38 \pm 12.78 \mathrm{a}$ \\
\hline
\end{tabular}

*Letras diferentes na linha significam diferença significativa entre médias.

Seis (12\%) dentre os 50 potros acompanhados apresentaram envolvimento sistêmico. Destes seis, um $(17 \%)$ pertencia ao grupo-controle e demonstrou comprometimento caracterizado por apatia discreta. Os cinco $(83 \%)$ outros pertenciam ao grupo-problema, dos quais um era pertencente ao grupo-problema degenerativo, com apatia discreta, e quatro ao grupo-problema inflamatório, demonstrando discreto quadro de letargia (2), asfixia moderada e eliminação de mecônio intrauterina (1) e asfixia severa, evoluindo para o óbito (1). 
Na avaliação hematológica (Tab. 3), todos os grupos mantiveram o HT e as PPT dentro dos valores de referência e sem diferenças entre os grupos. Com relação ao FB, o grupo-problema inflamatório apresentou valores mais altos, acima de $500 \mathrm{mg} / \mathrm{dL}$. Na contagem de LT, todas as médias foram mais baixas que os valores de referência.

$\mathrm{Na}$ avaliação bioquímica, os valores de GGT foram significativamente elevados no grupoproblema com relação ao grupo-controle $(\mathrm{P}<0,02)$, ultrapassando os limites de referência. No grupo-problema inflamatório também ocorreu elevação significativa do GGT com relação ao do grupo-controle $(\mathrm{P}<0,01)$. A média de AST para o grupo-problema também esteve acima dos limites de referência, com elevação significativa com relação ao grupo-controle $(\mathrm{P}<0,01)$, sendo esta situação repetida na comparação do grupo-problema degenerativo com o grupo-controle $(\mathrm{P}<0,01)$. As taxas de ureia foram elevadas em todos os grupos, mas o aumento só foi significativo no grupo-problema inflamatório $(\mathrm{P}<0,05)$ em relação ao grupocontrole. Creatinina apresentou médias semelhantes entre os quatro grupos, e os valores de bilirrubina foram semelhantes entre os grupos e se mantiveram dentro dos valores de referência.

Os valores hematológicos e bioquímicos estão demonstrados na Tab. 3 .

Tabela 3. Médias e desvio-padrão dos valores hematológicos e bioquímicos dos potros nos diferentes grupos

\begin{tabular}{lcccc} 
& Grupo-controle & Grupo-problema & $\begin{array}{c}\text { Grupo-problema } \\
\text { inflamatório }\end{array}$ & $\begin{array}{c}\text { Grupo-problema } \\
\text { degenerativo }\end{array}$ \\
\cline { 2 - 5 } & Média & Média & Média & Média \\
\hline HT $(\%)$ & $42.00 \pm 1.05 \mathrm{a}$ & $43.00 \pm 0.99 \mathrm{a}$ & $43.00 \pm 1.92 \mathrm{a}$ & $43.00 \pm 1.21 \mathrm{a}$ \\
PPT $(\mathrm{g} / \mathrm{dL})$ & $6.44 \pm 0.17 \mathrm{a}$ & $6.37 \pm 0.17 \mathrm{a}$ & $6.60 \pm 0.30 \mathrm{a}$ & $6.28 \pm 0.20 \mathrm{a}$ \\
FB $(\mathrm{mg} / \mathrm{dL})$ & $370.59 \pm 36-37 \mathrm{a}$ & $380.00 \pm 33.54 \mathrm{a}$ & $540.00 \pm 71.23 \mathrm{~b}$ & $326.67 \pm 27.67 \mathrm{a}$ \\
LT $\left(\times 10^{3} / \mu \mathrm{L}\right)$ & $5.33 \pm 0.32 \mathrm{a}$ & $5.72 \pm 0.32 \mathrm{a}$ & $5.00 \pm 0.77 \mathrm{a}$ & $5.86 \pm 0.36 \mathrm{a}$ \\
GGT $(\mathrm{U} / \mathrm{L})$ & $27.33 \pm 8.20 \mathrm{a}$ & $57.50 \pm 8.70 \mathrm{~b}$ & $34.00 \pm 10.64$ & $65.33 \pm 9.75 \mathrm{~b}$ \\
AST $(\mathrm{U} / \mathrm{L})$ & $195.80 \pm 29.87 \mathrm{a}$ & $318.88 \pm 28.92 \mathrm{~b}$ & $266.75 \pm 49.57 \mathrm{a}$ & $336.25 \pm 33.13 \mathrm{~b}$ \\
Ureia $(\mathrm{mg} / \mathrm{dL})$ & $28.78 \pm 3.36 \mathrm{a}$ & $38.19 \pm 3.56 \mathrm{a}$ & $46.00 \pm 7.19 \mathrm{~b}$ & $35.58 \pm 4.17 \mathrm{a}$ \\
Creatinina $(\mathrm{mg} / \mathrm{dL})$ & $1.41 \pm 0.25 \mathrm{a}$ & $1.92 \pm 0.28 \mathrm{a}$ & $2.33 \pm 0.56 \mathrm{a}$ & $1.82 \pm 0.30 \mathrm{a}$ \\
Bilirrubina $(\mathrm{mg} / \mathrm{dL})$ & $1.64 \pm 2.05 \mathrm{a}$ & $1.00 \pm 0.32 \mathrm{a}$ & $1.00 \pm 0.86 \mathrm{a}$ & $1.00 \pm 0.47 \mathrm{a}$ \\
\hline
\end{tabular}

HT: hematócrito; PPT: proteínas plasmáticas totais; FB: fibrinogênio; LT: leucócitos totais; GGT: gamaglutamiltransferase; AST: aspartato aminotransferase.

*Letras diferentes na linha significam diferença significativa entre médias.

\section{DISCUSSÃO}

Neste estudo, foi realizada a avaliação da placenta por meio do exame histológico para a identificação e caracterização de lesões que comprometam as membranas fetais, correlacionando estes achados com as avaliações clínica e metabólica dos potros neonatos. Não foram observadas diferenças no peso da placenta ou na relação do peso da placenta com o peso do potro entre os grupos. É descrito que a relação do peso da placenta com o do potro é um indicativo de placentite, porque, na presença da condição, ocorre edema da placenta, e este pode ser o principal fator no aumento do peso. Entretanto, neste experimento, a relação do peso da placenta com o peso do potro no pós-parto não foi superior a $11 \%$, o que é considerado normal segundo Knottembelt et al. (2004).
O peso dos potros do grupo-problema inflamatório foi significativamente menor que o peso dos outros grupos. Para Bain (2004), casos de placentite podem desencadear um parto prematuro com a produção de um potro debilitado. O baixo peso ao nascimento parece ser um reflexo de uma falha no processo de nutrição placentária, causando um subdesenvolvimento fetal, mesmo nas gestações a termo. Isso porque os portos nascidos das placentas com lesões inflamatórias, além do menor peso, apresentaram debilidade de comportamento, observada pelo maior tempo em se posicionar em decúbito esternal e para levantar após o parto.

O exame hematológico fornece importantes dados para a avaliação clínica dos potros neonatos. O número de leucócitos pode estar 
reduzido em situações de imaturidade ou sepse. A taxa de fibrinogênio é baixa em neonatos e níveis elevados indicam que os potros sofreram algum desafio dentro do útero (Morresey, 2005). Os dados deste estudo mostraram aumento do fibrinogênio nos potros nascidos de éguas que produziram placentas com alterações inflamatórias, o que pode ser indicativo de resposta intra-uterina destes potros ao processo inflamatório da placenta.

A avaliação da bioquímica sérica é útil em identificar potros de risco. Para Franklin (2007), algumas variáveis bioquímicas são indicativo de hipóxia tecidual localizada, como elevação na concentração das enzimas musculares (CPK e AST), hepáticas (GGT) e metabólitos hepáticos (bilirrubina) e renais (creatinina e ureia). No presente estudo, foi observado um aumento da atividade enzimática de AST e GGT nos potros nascidos de placenta com alterações degenerativas.

Existe uma inter-relação entre a placenta e o fígado fetal que é essencial no fornecimento de glutamina e asparagina para o metabolismo do feto, já que ambos os aminoácidos são transferidos da circulação materna para o feto através da placenta. Sua conversão hepática em glutamato e aspartato, bem como o recebimento e a oxidação destes produtos pela placenta, ajuda a regular as concentrações plasmáticas destes aminoácidos no feto (Hay Jr., 1995). Em éguas, a placenta é responsável pelo fornecimento de glutamina para o metabolismo fetal (Manso Filho et al., 2009). A insuficiência uteroplacentária resulta no comprometimento das trocas metabólicas e gasosas entre a mãe e o feto, causando redução na oferta de aminoácidos pela placenta, associada à maior utilização destes pelo feto por meio do aumento da oxidação mitocondrial (Marconi et al., 1999). Entretanto, os animais do presente experimento não tiveram comprometimento clínico, mas provavelmente alguma alteração subclínica evidenciada pelo aumento dos níveis enzimáticos, o que sugere a eficácia do processo metabólico adaptativo.

Níveis elevados de creatinina e ureia sérica foram descritos em humanos recém-nascidos sofrendo de asfixia perinatal, devido à alta sensibilidade do rim a uma redução no aporte de oxigênio (Gupta et al., 2005). Para Vaala (1999),
Bernard (2003) e Morresey (2005), o nível de creatinina sérica não é um marcador confiável da função renal em potros neonatos, porque a placenta é a principal responsável pela eliminação de metabólitos do feto, e a elevação da creatinina é geralmente uma consequência de disfunção placentária. No presente estudo, os níveis de ureia foram superiores aos valores de referência em todos os grupos, sendo que houve uma elevação significativa nos potros nascidos de placentas com alterações inflamatórias em relação ao grupo- controle. Já os níveis de creatinina se mantiveram dentro dos limites de referência e sem diferenças entre os grupos, demonstrando que não houve oscilação deste metabólito, mesmo em situações de insuficiência placentária. Desta forma, apesar dos níveis estáveis de creatinina, os potros do grupoproblema inflamatório podem ter sofrido algum grau de comprometimento renal em razão da elevação significativa nas taxas de ureia, porque este metabólito é melhor indicativo de função renal.

Além da elevação significativa nos níveis de ureia no grupo-problema inflamatório, no grupoproblema, a ureia apresentou uma elevação com tendência a ser significativa $(\mathrm{P}<0,07)$, sugerindo que os níveis de ureia têm uma relação direta com situações de insuficiência placentária e comprometimento renal. Desta forma, a ureia pode ser um marcador em potencial para determinar insuficiência renal em potros neonatos, decorrente de lesão placentária.

\section{CONCLUSÕES}

O exame histopatológico da placenta apresenta resultados compatíveis com a apresentação clínica do potro. A ocorrência de lesões inflamatórias na placenta está diretamente relacionada ao comprometimento clínico do potro. Já as lesões placentárias degenerativas não estão associadas ao comprometimento clínico severo dos potros, mas podem ser responsáveis pela manifestação de distúrbios subclínicos, evidenciados pelo aumento das taxas de AST e GGT. Pode-se concluir que a ureia é um indicador de comprometimento renal decorrente de insuficiência placentária em potros neonatos, podendo ser explorada como um marcador desta condição. 


\section{REFERÊNCIAS}

ALLEN, T.C. Hematoxilin and eosin. In: PROPHET, E.B.; MILLS, B.; ARRINGTON, J.B.; SOBIN, L.H. (ED). Laboratory Methods in Histotechnology - Armed Forces Institute of Pathology. Washington: American Registry of Pathology, 1994. p.53-57.

BAIN, F.T. Management of the foal from the mare with placentitis: A clinician's approach. In: ANNUAL CONVENTION OF THE AMERICAN ASSOCIATION OF EQUINE PRACTITIONERS, 50., 2004, Denver-CO. Proceedings... Ithaca: International Veterinary Information Service (www.ivis.org), 2004. Doc. n. P1419.1204.

BERNARD, W.V. Jump-starting the dummy foal (Neonatal Maladjustment Syndrome/Hypoxic Ischemic Encephalopathy). Proc. Am. Assoc. Equine Pract., v.49, p.8-12, 2003.

FRANKLIN, R.P. Identification and treatment of the high-risk foal. Proc. Am. Assoc. Equine Pract., v.53, p.320-328, 2007.

GUPTA, B.D.; SHARMA, P.; BAGLA, J. et al. Renal failure in asphyxiated neonates. Ind. Pediat., v.42, p.928-934, 2005.

HAY Jr., W.W. Current topic: Metabolic interrelationships of placenta and fetus. Plac., v.16, p.19-30, 1995.
HONG, C.B.; DONAHUE, J.M.; GILES Jr., R.C. et al. Etiology and pathology of equine placentitis. J. Vet. Diag. Invest., v.5, p.56-63, 1993.

JANSSON, T.; POWELL, T.L. Human placental transport in altered fetal growth: does the placenta function as a nutrient sensor? A review. Plac., v.27, Suppl. A, p.91-97, 2006.

KNOTTENBELT, D.; HOLDSTOCK, N.; MADIGAN, J. Equine Neonatal Medicine and Surgery. Filadélfia:Saunders, 2004. 368p.

MANSO FILHO, H.C.; COSTA, H.E.; WU, G. et al. Equine placenta expresses glutamine synthetase. Vet. Res. Comm., v.33, p.175-182, 2009.

MARCONI, A.M.; PAOLINI, C.L.; STRAMARE, L. et al. Steady state maternal-fetal leucine enrichments in normal and intrauterine growthrestricted pregnancies. Pediat. Res., v.46, p.114$119,1999$.

MORRESEY, P.R. Prenatal and perinatal indicators os neonatal viability. Clin. Tech. Equine Pract., v.4, p.238-249, 2005.

SCHLAFER, D. Postmortem examination of the equine placenta, fetus, and neonate: Methods and interpretation of findings. Proc. Am. Assoc. Equine Pract., v.50, p.144-161, 2004.

VAALA, W.E. Peripartum asphyxia syndrome in foals. Proc. Am. Assoc. Equine Pract., v.45, p.247-253, 1999. 